\title{
Attitude Error Representations for Kalman Filtering
}

\author{
F. Landis Markley*
}

The quaternion has the lowest dimensionality possible for a globally nonsingular attitude representation. The quaternion must obey a unit norm constraint, though, which has led to the development of an extended Kalman filter using a quaternion for the global attitude estimate and a three-component representation for attitude errors. We consider various attitude error representations for this Multiplicative Extended Kalman Filter and its second-order extension.

\section{INTRODUCTION}

Use of the Extended Kalman Filter $(E K F)^{1-4}$ for spacecraft attitude estimation has a long history. The earliest application ${ }^{5}$ used three Euler angles to parameterize the attitude, and one of the most recent examples employs the rotation vector. ${ }^{6}$ Both of these parameterizations require transcendental function evaluations and are singular for certain attitudes, so other parameterizations have been employed for Kalman filtering. The search for an ideal parameterization is frustrated by the fact that a globally nonsingular threedimensional parameterization of the rotation group is topologically impossible. ${ }^{7}$

The unit quaternion has the lowest dimension of any globally nonsingular attitude parameterization and represents the attitude matrix as a homogeneous quadratic function. The kinematic propagation equation for the quaternion is bilinear in the components of the quaternion and the angular velocity vector. These advantages have motivated the development of filters using the quaternion representation for both the global attitude and the attitude errors. ${ }^{8}$ The linear measurement updates provided by these straightforward EKFs violate the nonlinear quaternion normalization constraint, however. Several solutions have been proposed for this problem, with varying degrees of success. ${ }^{8-12}$

In this paper, we will consider an EKF that parameterizes the global attitude with a unit quaternion, while employing a three-component representation for the attitude errors. ${ }^{9,13,14}$ Several justifications have been provided for this non-standard approach to Kalman filtering. The approach in Section IX of Ref. 9 regards the filter as estimating a four-component quaternion, but argues that the quaternion covariance is rankdeficient due to quaternion norm constraint, or equivalently due to the underlying three-dimensional nature of the rotation group. ${ }^{7,15,16}$ Since the $4 \times 4$ quaternion covariance is assumed to have rank three, it can be projected onto a $3 \times 3$ matrix without any loss of information. This gives a measurement update obeying the quaternion norm constraint to first order in the measurement residual, so enforcing exact quaternion normalization only modifies the quaternion to second order and is therefore outside the purview of the EKF. Vathsal ${ }^{17}$ has extended this approach to second order; but the result does not appear to be a consistent second-order filter, since his quaternion update violates the norm constraint in second order.

An alternative approach, which has become known as the multiplicative EKF (MEKF), is set forth in Section XI of Ref. 9. This regards the filter as estimating a three-component attitude error vector, which naturally has a $3 \times 3$ covariance, while parameterizing the global attitude by a quaternion. This is actually the original approach, ${ }^{13.14}$ and it leads to the same algorithm as the covariance projection approach in the linearized EKF approximition. It rests on a firmer conceptual basis, however.

This paper is an extension of Ref. 9, which provides citations of the literature through 1981. Its principal aims are to consider in some depth the relationship between the three-component attitude error vector and the quaternion, and to extend the MEKF approach to a consistent second-order Kalman filter. To set the stage, we begin with a brief discussion of attitude representations. We then develop the basic equations of the MEKF, and consider its extension to second order.

*Guidance, Navigation and Control Systems Engineering Branch, NASA's Goddard Space Flight Center, Greenbelt, MD 20771 


\section{ATTITUDE PARAMETERIZATIONS}

Good reviews of attitude representations are available. ${ }^{18,19}$ We provide a brief discussion to establish conventions and notation for the representations of interest to this paper. We regard the $3 \times 3$ orthogonal attitude matrix, or direction cosine matrix as the most fundamental representation of the attitude.

\section{Rotation Vector}

Euler's Theorem ${ }^{20}$ states that the most general motion of a rigid body with one point fixed is a rotation by an angle $\phi$ about some axis. We specify the axis by a unit vector $\mathbf{e}$ and combine the axis and angle into a rotation vector $\phi \equiv \phi \mathbf{e}$. The attitude matrix as a function of $\phi$ is

$$
A(\phi)=I_{3 \times 3}-(\sin \phi / \phi)[\phi \times]+\left[(1-\cos \phi) / \phi^{2}\right][\phi \times]^{2},
$$

where the $3 \times 3$ identity matrix is denoted by $I_{3 \times 3}$ and the cross product matrix is

$$
[\mathbf{q} \times] \equiv\left[\begin{array}{ccc}
0 & -q_{3} & q_{2} \\
q_{3} & 0 & -q_{1} \\
-q_{2} & q_{1} & 0
\end{array}\right] .
$$

Equation (1) and the kinematic equation for the rotation vector are transcendental and ill behaved, though finite, for zero rotation angle. All rotations can be mapped to points inside and on the surface of a sphere of radius $\pi$ in rotation vector space, where points at opposite ends of a diameter represent the same rotation; but the rotation vector may jump from one end of a diameter to the other as the attitude varies smoothly. These jumps can be postponed by expanding the representation to a sphere of radius $2 \pi$, but they cannot be avoided entirely, since the kinematic equation for the rotation vector is singular for $\phi=2 \pi$. These characteristics limit the usefulness of the rotation vector as a global attitude representation.

\section{Quaternions}

A unit quaternion representing spacecraft attitude has a three-vector part and a scalar part, which are related to the axis and angle of rotation by

$$
q=\left[\begin{array}{l}
q_{V} \\
q_{4}
\end{array}\right]=\left[\begin{array}{c}
\mathbf{q} \\
q_{4}
\end{array}\right]=\left[\begin{array}{c}
\mathbf{e} \sin (\phi / 2) \\
\cos (\phi / 2)
\end{array}\right],
$$

and obey the unit length constraint

$$
|q|^{2} \equiv|\mathbf{q}|^{2}+q_{4}^{2}=1
$$

The four components of $q$ are the Euler symmetric parameters or the Euler-Rodrigues parameters, which first appeared in a paper by Euler ${ }^{21}$ and in unpublished notes by Gauss. ${ }^{22}$ Rodrigues' classic paper of 1840 first demonstrated their general usefulness. ${ }^{23}$ Hamilton introduced the quaternion as an abstract mathematical object in $1844,{ }^{24}$ but there is some question as to whether he correctly understood its relation to rotations. ${ }^{25}$

Unit quaternions reside on the three-dimensional sphere $\$ 3$ embedded in four-dimensional Euclidean space E4. The attitude matrix a homogeneous quadratic function of the components of a unit quaternion;

$$
A(q)=\left(q_{4}^{2}-|\mathbf{q}|^{2}\right) I_{3 \times 3}-2 q_{4}[\mathbf{q} \times]+2 \mathbf{q} \mathbf{q}^{T} .
$$

The quaternion representation is $2-1$ because Eq. (5) shows that $q$ and $-q$ represent the same rotation matrix. The quaternion is an ideal global attitude representation, since it varies continuously over $\mathrm{S} 3$ as the attitude changes, avoiding the jumps required by some three-dimensional parameterizations. It is customary to restrict a quaternion representing an attitude error to the hemisphere of S3 with $q_{4}>0$, however. 
We follow Ref. 19 in writing the quaternion product as

$$
p \otimes q \equiv\left[\begin{array}{c}
p_{4} \mathbf{q}+q_{4} \mathbf{p}-\mathbf{p} \times \mathbf{q} \\
p_{4} q_{4}-\mathbf{p} \cdot \mathbf{q}
\end{array}\right] .
$$

This differs from the historical multiplication convention, ${ }^{18.24}$ denoted by $q p$ without an infix operator, by the sign of the cross product in the vector part. The two products are related by $p \otimes q=q p$. The convention adopted here has the useful property that

$$
A(p) A(q)=A(p \otimes q),
$$

as contrasted with $A(p) A(q)=A(q p)$. Equation (7) means that the rotation group and the quaternion group are almost isomorphic, the qualifier "almost" owing to the 2-1 nature of the mapping. ${ }^{16}$ We use an overbar to denote the quaternion representation of a three-vector.

$$
\overline{\mathbf{v}} \equiv\left[\begin{array}{l}
\mathbf{v} \\
0
\end{array}\right] .
$$

With this convention, the kinematic equation for the quaternion can be written in the alternative forms

$$
\dot{q}=\frac{1}{2} \overline{\boldsymbol{\omega}} \otimes q=\frac{1}{2} \Omega(\boldsymbol{\omega}) q,
$$

where $\omega$ is the angular velocity vector, and the skew-symmetric $4 \times 4$ matrix $\Omega(\omega)$ is defined by

$$
\Omega(\boldsymbol{\omega}) \equiv\left[\begin{array}{cc}
-[\omega \times] & \omega \\
-\omega^{T} & 0
\end{array}\right] .
$$

The skew-symmetry of $\Omega(\omega)$ preserves the normalization of $q$, but this normalization may be lost due to computational errors. If so, it can be restored trivially by $q=q /|q|$.

\section{Gibbs Vector or Rodrigues Parameters}

The three Rodrigues parameters are defined by ${ }^{23}$

$$
\mathbf{g} \equiv \frac{\mathbf{q}}{q_{4}}=\frac{\mathbf{e} \sin (\phi / 2)}{\cos (\phi / 2)}=\mathbf{e} \tan (\phi / 2)
$$

Gibbs arrayed them in a vector semitangent of version; it is little wonder that we now call it the Gibbs vector. ${ }^{26}$ The Gibbs vector can be regarded as a gnomonic projecton of the S3 quaternion space onto threedimensional Euclidean $\mathbf{g}$ space, as shown in Fig. 1. This is a 2-1 mapping of S3, with $q$ and $-q$ mapping to the same point. Since $q$ and $-q$ represent the same rotation, the Gibbs vector parameterization is a 1-1 representation of the rotations onto E3. The Gibbs vector is infinite for $180^{\circ}$ rotations (the $q_{4}=0$ equator of $\$ 3$ ), which is undesirable for a global representation of rotations. The attitude matrix has the Gibbs vector representation

$$
A(\mathbf{g})=I_{3 \times 3}-2\left(1+g^{2}\right)^{-1}\left\{[\mathbf{g} \times]-[g \times]^{2}\right\},
$$

where italics are used to denote the magnitude of any three-vector other than the vector part of a quaternion.

\section{Modified Rodrigues Parameters}

These parameters were introduced by Wiener: ${ }^{27}$

$$
\mathbf{p} \equiv \frac{\mathbf{q}}{1+q_{4}}=\frac{\mathbf{e} \sin (\phi / 2)}{1+\cos (\phi / 2)}=\mathbf{e} \tan (\phi / 4),
$$


and represent the attitude matrix by

$$
A(\mathbf{p})=I_{3 \times 3}-4\left(1+p^{2}\right)^{-2}\left\{\left(1-p^{2}\right)[\mathbf{p} \times]-2[\mathbf{p} \times]^{2}\right\} .
$$

Marandi and Modi ${ }^{28}$ pointed out that the Modified Rodrigues Parameters can be viewed as a stereographic projection of S3 quaternion space, as shown in Fig. 2. One hemisphere of S3 projects to the interior of the unit sphere in three-dimensional $\mathbf{p}$ space, and the other hemisphere of $\mathrm{S} 3$ projects to the exterior of the unit p-sphere. All rotations can be represented by Modified Rodrigues Parameters inside and on the surface of the unit ball. If we extend the representation to all Euclidean $\mathbf{p}$ space, we have a $2-1$ parameterization with $\mathbf{p}$ and $-\mathbf{p} / \mathbf{p}^{2}$ representing the same rotation. This parameterization shares many characteristics with the rotation vector parameterization, including the need for discrete jumps, but avoids transcendental functions.

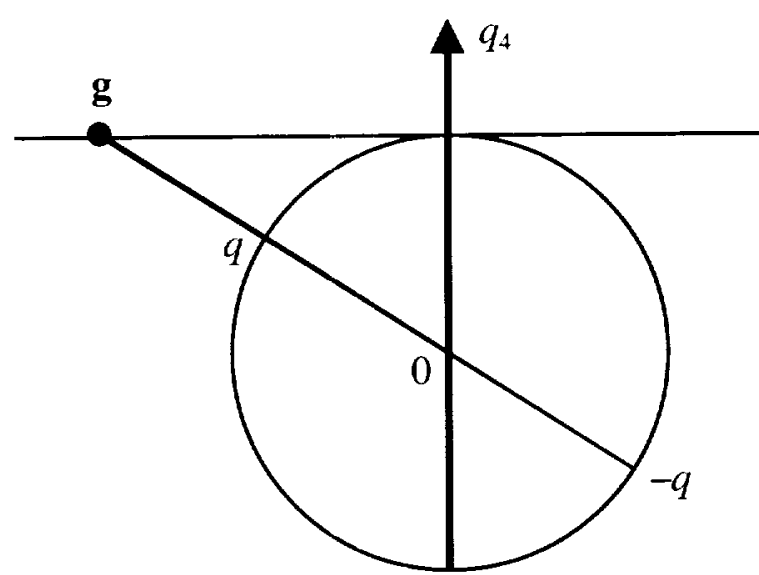

Figure L. Gibbs Vector as a Gnomonic Projection

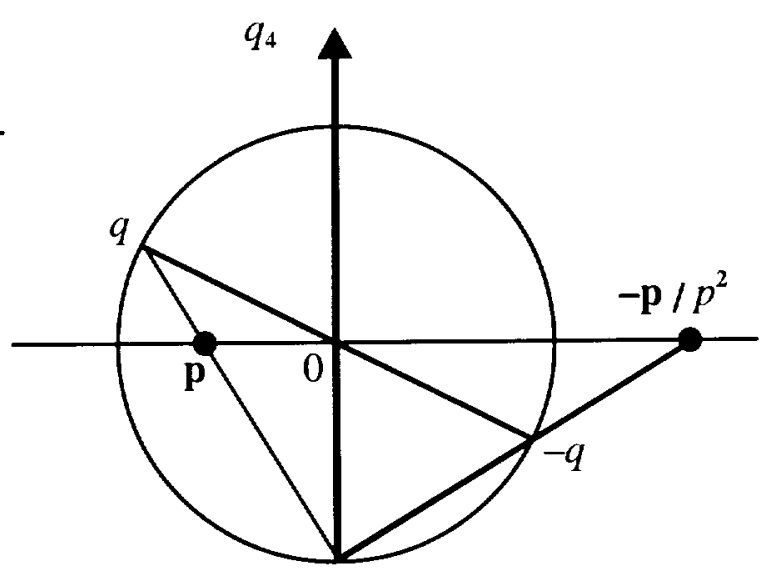

Figure 2. Modified Rodrigues Parameters as a Stereographic Projection

\section{MULTIPLICATIVE EXTENDED KALMAN FILTER}

The MEKF represents the true nonlinear state as the quaternion product

$$
q(t)=\delta q(\mathbf{a}(t)) \otimes q_{r e f}(t)
$$

where $q_{r e f}(t)$ is some unit reference quaternion and $\delta q(\mathbf{a}(t))$ is a unit quaternion representing the rotation from $q_{r e f}(t)$ to the true attitude parameterized by $q(t)$. We parameterize $\delta q(\mathbf{a}(t))$ by a three-vector $\mathbf{a}(t)$ of attitude errors in the spacecraft body frame. The two attitude representations $\mathbf{a}(t)$ and $q_{\text {ref }}(t)$ in Eq. (15) are clearly redundant. The MEKF computes an unconstrained estimate of the three-component a(t) while using the four-component $q_{r, f}(t)$ to provide a globally nonsingular attitude representation. An alternative formulation, which has some advantages, reverses the order of multiplication in Eq. (15) so that $\mathbf{a}(t)$ represents the attitude errors in the inertial reference frame rather than the errors in the body frame. ${ }^{29}$

Given an estimate of the error vector $\hat{\mathbf{a}}(t)$, the best estimate of the attitude quaternion is

$$
\hat{q}(t) \equiv \delta q(\hat{\mathbf{a}}(t)) \otimes q_{r e f}(t),
$$

which is a unit quaternion. We remove the redundancy in the attitude representation by choosing the reference quaternion $q_{r, j}(t)$ so that the expectation value $\hat{\mathbf{a}}(t)$ is identically zero when all available dynamic 
and measurement information is included. Then Eq. (16) shows that $q_{r e f}(t)$ is identically equal to $\hat{q}(t)$, so we will denote it as such. This means in turn that $\delta q(\mathbf{a}(t))$ is a representation of the attitude error. It is important to note that if $\hat{q}(t)$ had been defined as the expectation of some probability distribution over S3, it would not be a unit quaternion unless the distribution were concentrated at a point, since restricting the probability distribution in quaternion space to the surface of $\mathrm{S} 3$ means that its expectation value must be inside S3. This is the conceptual advantage of the MEKF.

Continuous/discrete filtering proceeds in three steps: time propagation, measurement update, and reset. The continuous time propagation is arranged to keep $\hat{\mathbf{a}}(t) \equiv \mathbf{0}$, but the discrete measurement update assigns a finite post-update value $\hat{\mathbf{a}}(+)$ to $\hat{\mathbf{a}}$. In order to avoid the need to propagate two representations of the attitude, the reset operation moves the attitude information from $\hat{\mathbf{a}}(+)$ to $\hat{q}(+)$, after which $\hat{\mathbf{a}}$ is reset to zero. Since true quaternion is not changed by this operation, Eq. (15) requires

$$
\delta q(\hat{\mathbf{a}}(+)) \otimes \hat{q}(-)=\delta q(\mathbf{0}) \otimes \hat{q}(+)=\hat{q}(+),
$$

where $\hat{q}(-)$ is the pre-update quaternion. It is possible eliminate the discrete reset by keeping $\hat{\mathbf{a}}(t) \equiv \mathbf{0}$ at all times, even during the update, by considering each attitude measurement update to be spread out over an infinitesimal time interval, rather than being instantaneous. ${ }^{30}$ This paper treats measurement updates as discrete rather than continuous, however.

The significance of the reset is obscured in the standard EKF, which represents the true state $\mathbf{X}$ as the sum of the reference value $\hat{\mathbf{X}}$ and a small error $\mathbf{x}$,

$$
\mathbf{X}=\hat{\mathbf{X}}+\mathbf{x}
$$

The measurement processing produces an updated value of the error vector

$$
\hat{\mathbf{x}}(+)=\hat{\mathbf{x}}(-)+\Delta \mathbf{x} .
$$

The reset operation moves the update information from the error state to the estimate of the full state by

$$
\hat{\mathbf{X}}(+)=\hat{\mathbf{X}}(-)+\hat{\mathbf{x}}(+)-\hat{\mathbf{x}}(-)=\hat{\mathbf{X}}(-)+\Delta \mathbf{x}
$$

which gives the appearance that the update is applied directly to the full state estimate. In fact, an implicit reset of the gyro biases is preformed in exactly this manner in the filter considered later in this paper. The reset of the attitude must be treated explicitly in the MEKF, however.

\section{Attitude Error Representations}

One possible parameterization of $\mathbf{a}$ is the rotation vector $\phi \mathbf{e}$, which we denote $\mathbf{a}_{\phi}$, so from Eq. (3),

$$
\delta q\left(\mathbf{a}_{\phi}\right)=\left[\begin{array}{c}
\left(\mathbf{a}_{\phi} / a_{\phi}\right) \sin \left(a_{\phi} / 2\right) \\
\cos \left(a_{\phi} / 2\right)
\end{array}\right] .
$$

This parameterization has the advantage that the covariance includes the angular variances in radians ${ }^{2}$, but it is numerically inconvenient owing to the appearance of trigonometric functions and $\mathbf{a}_{\phi} / a_{\phi}$, which is indeterminate at $a_{\phi}=0$.

We can retain this interpretation of the covariance matrix by requiring a to be equal to the rotation vector in the small angle approximation. This leads to the second parameterization of a as twice the vector part of $\delta q$, which is the parameterization used in Section XI of Ref. 9, except for the factor of two;

$$
\delta q\left(\mathbf{a}_{q}\right)=\frac{1}{2}\left[\frac{\mathbf{a}_{q}}{\sqrt{4-a_{q}^{2}}}\right] .
$$


A third alternative is four times the vector of Modified Rodrigues Parameters, so from Eq. (13),

$$
\delta q\left(\mathbf{a}_{p}\right)=\frac{1}{16+a_{p}^{2}}\left[\begin{array}{c}
8 \mathbf{a}_{p} \\
16-a_{p}^{2}
\end{array}\right] .
$$

This parameterization has the computational advantage of not requiring any transcendental functions.

A fourth parameterization is twice the Gibbs vector; from the inverse of Eq. (11),

$$
\delta q\left(\mathbf{a}_{g}\right)=\frac{1}{\sqrt{4+a_{g}^{2}}}\left[\begin{array}{c}
\mathbf{a}_{g} \\
2
\end{array}\right] .
$$

The Gibbs vector form has the advantage that the reset can first define the unnormalized quaternion

$$
\rho \equiv\left[\begin{array}{c}
\mathbf{a}_{s} \\
2
\end{array}\right] \otimes \hat{q}(-)
$$

and then the updated unit quaternion is given by

$$
\hat{q}(+)=\rho /|\rho|
$$

This form avoids any accumulation of numerical errors in the quaternion norm.

These four definitions of a provide the same second-order approximations to the quaternion,

$$
\delta q(\mathbf{a})=\left[\begin{array}{c}
\mathbf{a} / 2 \\
1-a^{2} / 8
\end{array}\right],
$$

and to the attitude matrix

$$
A(\delta q)=I_{3 \times 3}-[\mathbf{a} \times]+\frac{1}{2}\left(\mathbf{a a}^{T}-a^{2} I_{3 \times 3}\right) .
$$

Thus they are equivalent for an EKF, which uses a linear approximation; but they differ in third and higher orders in a. It is worthwhile to note that Eqs. (24) and (25) only hold to first order if the components of a are taken to be Euler angle rotations about three orthogonal axes, as in Refs. 5 and 13. Thus, an Euler angle parameterization will lead to the same EKF, but will give different results for a second order filter.

The error representations are quite different for large errors, which may arise before the Kalman filter has converged. Consider the largest possible attitude error, which is a $180^{\circ}$ rotation. This corresponds in the various three-component representations to $a_{i q}=2, a_{\phi}=\pi, a_{p}=4$ or infinite $a_{g}$. A filter update $\hat{\mathbf{a}}(+)$ larger than this value does not give a sensible quaternion reset. The infinite limiting value for the Gibbs vector representation is another reason to prefer this representation.

\section{State and Covariance Propagation}

The filter dynamics are found by differentiating

$$
\delta q(t)=q(t) \otimes \hat{q}^{-1}(t),
$$

using Eq. (9) and the identity

$$
\mathrm{d} \hat{q}^{-1} / \mathrm{d} t=-\hat{q}^{-1} \otimes \dot{\hat{q}} \otimes \hat{q}^{-1}
$$

This follows, like the corresponding expression for the derivative of the matrix inverse, from the vanishing of the time derivative of $\hat{q} \otimes \hat{q}^{-1}$, which is the constant identity quaternion. This gives

$$
\mathrm{d}(\delta \mathrm{q}(\mathbf{a})) / \mathrm{d} t=\frac{1}{2} \overline{\mathbf{\omega}} \otimes \delta q(\mathbf{a})-\delta q(\mathbf{a}) \otimes \dot{\hat{q}} \otimes \hat{q}^{-1} .
$$


Taking the expectation value of this equation in the usual linear EKF approximation of ignoring correlations gives

$$
\mathrm{d}(\delta \mathrm{q}(\hat{\mathbf{a}})) / \mathrm{d} t=\frac{1}{2} \overline{\hat{\mathbf{\omega}}} \otimes \delta q(\hat{\mathbf{a}})-\delta q(\hat{\mathbf{a}}) \otimes \dot{\hat{q}} \otimes \hat{q}^{-1}
$$

Since $\delta q(\hat{\mathbf{a}})$ is the identity quaternion, which is a constant, we have

$$
0=\frac{i}{2} \overline{\hat{\boldsymbol{\omega}}}-\dot{\hat{q}} \otimes \hat{q}^{-1}
$$

or

$$
\dot{\hat{q}}(t)=\frac{1}{2} \overline{\hat{\boldsymbol{\omega}}}(t) \otimes \hat{q}(t)=\frac{1}{2} \Omega(\hat{\boldsymbol{\omega}}(t)) \hat{q}(t),
$$

This quaternion propagation equation is the same as the equation derived by more conventional methods, but we have derived it from the requirement that $\hat{\mathbf{a}}(t)$ be identically zero.

Now we specialize to the case where a set of gyros is used to obtain angular rate information in place of models of the spacecraft dynamics. The Kalman filter estimates the spacecraft attitude and a three-vector of gyro drift biases. ${ }^{9,14}$ We employ Farrenkopf's gyro dynamics error model, ${ }^{31}$ which means that we ignore the output noise for rate-inlegrating gyros. ${ }^{32}$ This is an excellent approximation for navigation-grade gyros.

The angular rate vector is given in terms of the gyro output vector $\tilde{\boldsymbol{\omega}}(t)$ and gyro drift vector $\mathbf{b}(t)$ in spacecraft body coordinates by

$$
\boldsymbol{\omega}(t)=\tilde{\omega}(t)-\mathbf{b}(t)-\eta_{1}(t)
$$

where $\eta_{1}(t)$ is a zero-mean white noise process. The gyro drift vector obeys

$$
\dot{\mathbf{b}}(t)=\eta_{2}(t),
$$

where $\eta_{2}(t)$ is an independent zero-mean white noise process. This clearly implies that

$$
\dot{\hat{\mathbf{b}}}(t)=\mathbf{0}
$$

We consider the Gibbs vector parameterization for specificity. Solving Eq. (21d) for $\mathbf{a}_{g}$ gives

$$
\mathbf{a}_{g}(t)=2(\delta q)_{V} /(\delta q)_{4}
$$

where the subscript $V$ and 4 denote the vector and scalar parts of the quaternion, as in Eq. (3). Then, with Eqs. (15) and (28),

$$
\begin{aligned}
\mathbf{f}(\mathbf{x}(t), t) \equiv \dot{\mathbf{a}}_{g}(t)= & \left\{\frac{1}{2} \overline{\mathbf{\omega}}(t) \otimes\left[\begin{array}{c}
\mathbf{a}_{g}(t) \\
2
\end{array}\right]-\left[\begin{array}{c}
\mathbf{a}_{g}(t) \\
2
\end{array}\right] \otimes \dot{\hat{q}}(t) \otimes \hat{q}^{-1}(t)\right\}_{v} \\
& -\frac{1}{2}\left\{\frac{1}{2} \overline{\mathbf{\omega}}(t) \otimes\left[\begin{array}{c}
\mathbf{a}_{g}(t) \\
2
\end{array}\right]-\left[\begin{array}{c}
\mathbf{a}_{g}(t) \\
2
\end{array}\right] \otimes \dot{\hat{q}}(t) \otimes \hat{q}^{-1}(t)\right\}_{4} \mathbf{a}_{g}(t),
\end{aligned}
$$

where the time dependence of the reference quaternion is implicitly included in the time argument of $\mathbf{f}(\mathbf{x}(t), t)$. Inserting Eq. (31) gives, in the EKF approximation

$$
\mathbf{f}(\mathbf{x}(t), t)=-\hat{\boldsymbol{\omega}} \times \mathbf{a}_{g}(t)+\Delta \boldsymbol{\omega}(t)-\frac{1}{2} \Delta \boldsymbol{\omega}(t) \times \mathbf{a}_{g}(t)+\frac{1}{4}\left[\Delta \boldsymbol{\omega}(t) \cdot \mathbf{a}_{g}(t)\right] \mathbf{a}_{g}(t),
$$

where

$$
\Delta \boldsymbol{\omega}(t) \equiv \boldsymbol{\omega}(t)-\hat{\boldsymbol{\omega}}(t)=\boldsymbol{\omega}(t)-[\tilde{\boldsymbol{\omega}}(t)-\hat{\mathbf{b}}(t)]=\hat{\mathbf{b}}(t)-\mathbf{b}(t)-\eta_{1}(t)
$$


We want to construct an EKF for the six-component state vector

$$
\mathbf{x}(t) \equiv\left[\begin{array}{l}
\mathbf{a}(t) \\
\mathbf{b}(t)
\end{array}\right] .
$$

where we suppress the subscript on $\mathbf{a}$ in equations that hold for any three-dimensional parameterization. This state vector obeys the propagation equation

$$
\dot{\mathbf{x}}(t)=\left[\begin{array}{c}
\dot{\mathbf{a}} \\
\dot{\mathbf{b}}
\end{array}\right]=\left[\begin{array}{c}
\mathbf{f}(\mathbf{x}(t), t) \\
\eta_{2}(t)
\end{array}\right]
$$

The expectation value of the state vector was shown above to be constant in the absence of measurements.

The covariance matrix can be partitioned into $3 \times 3$ submatrices as

$$
P(t) \equiv E\left\{(\mathbf{x}-\hat{\mathbf{x}})(\mathbf{x}-\hat{\mathbf{x}})^{T}\right\}=\left[\begin{array}{cc}
P_{a}(t) & P_{r}(t) \\
P_{c}^{T}(t) & P_{b}(t)
\end{array}\right],
$$

We let $\partial \mathbf{f} / \partial \mathbf{a}$ denote the matrix with elements

$$
[\partial \mathbf{f} / \partial \mathbf{a}]_{i j} \equiv \partial f_{i} / \partial a_{j}
$$

and define the matrices

$$
\begin{gathered}
F(t) \equiv\left[\begin{array}{cc}
\mathrm{E}\{\partial \mathbf{f} / \partial \mathbf{a}\} & \mathrm{E}\{\partial \mathbf{f} / \partial \mathbf{b}\} \\
0_{3 \times 3} & 0_{3 \times 3}
\end{array}\right]=\left[\begin{array}{cc}
-[\hat{\boldsymbol{\omega}}(t) \times] & -I_{3 \times 3} \\
0_{3 \times 3} & 0_{3 \times 3}
\end{array}\right], \\
G(t) \equiv\left[\begin{array}{cc}
\mathrm{E}\left\{\partial \mathbf{f} / \partial \boldsymbol{\eta}_{1}\right\} & \mathrm{E}\left\{\partial \mathbf{f} / \partial \boldsymbol{\eta}_{2}\right\} \\
0_{3 \times 3} & I_{3 \times 3}
\end{array}\right]=\left[\begin{array}{cc}
-I_{3 \times 3} & 0_{3 \times 3} \\
0_{3 \times 3} & I_{3 \times 3}
\end{array}\right] .
\end{gathered}
$$

Then the time propagation of the covariance is given by

$$
\dot{P}(t)=F(t) P(t)+P(t) F^{T}(t)+G(t) Q(t) G^{T}(t)-P(t) H^{T}(t) R^{-1}(t) H(t) P(t),
$$

where

$$
Q(t)=\left[\begin{array}{cc}
Q_{1}(t) & 0_{3 \times 3} \\
0_{3 \times 3} & Q_{2}(t)
\end{array}\right]
$$

with

$$
\mathrm{E}\left\{\boldsymbol{\eta}_{i}(t) \boldsymbol{\eta}_{j}^{T}\left(t^{\prime}\right)\right\}=\delta_{i j} \delta\left(t-t^{\prime}\right) Q_{i}(t) \text { for } i, j=1,2
$$

The covariance propagation during the intervals between the attitude measurement updates is identical to Refs. 13 and 14, except for factors of $\frac{1}{2}$ in some of the formulations. The Appendix shows that these equations also hold if $\mathrm{Eq} .(2 \mathrm{lb})$ is used in place of Eq. (21d). By implication, these relations are independent of the representation chosen for a, as long as Eq. (24) is satisfied.

\section{Measurement Model and Update}

A measurement is modeled as a an $m$-dimensional function $\mathbf{h}$ of a vector $\mathbf{v}_{B}$ in the spacecraft body frame, with white noise added;

$$
\mathbf{z}=\mathbf{h}\left(\mathbf{v}_{B}\right)+\text { white noise }
$$


The representation of $\mathbf{v}_{B}$ in the body frame is the mapping of its representation $\mathbf{v}_{l}$ in the inertial reference frame by the attitude matrix:

$$
\mathbf{v}_{B}=A(q) \mathbf{v}_{l}=\left(I_{3 \times 3}-[\mathbf{a} \times]\right) \hat{\mathbf{v}}_{B},
$$

where we have used Eqs. (7), (15), and the first-order approximation to Eq. (24), and where $\hat{\mathbf{v}}_{B} \equiv A(\hat{q}) \mathbf{v}_{l}$ is the vector in the body frame that is predicted by the reference quaternion. This gives, to first order in $\mathbf{a}$,

$$
\mathbf{h}\left(\mathbf{v}_{B}\right)=\mathbf{h}\left(\hat{\mathbf{v}}_{B}\right)-\left.(\partial \mathbf{h} / \partial \mathbf{v})\right|_{\hat{v}_{B}}[\mathbf{a} \times] \hat{\mathbf{v}}_{B}=\hat{\mathbf{h}}+H_{a} \mathbf{a},
$$

where

$$
\hat{\mathbf{h}} \equiv \mathbf{h}\left(\hat{\mathbf{v}}_{B}\right)
$$

and

$$
\left.H_{a} \equiv(\partial \mathbf{h} / \partial \mathbf{v})\right|_{\hat{\mathbf{v}}_{u}}\left[\hat{\mathbf{v}}_{B} \times\right] .
$$

Thus the $m \times 6$ measurement sensitivity matrix is

$$
H=[\partial \mathbf{h} / \partial \mathbf{a} \quad \partial \mathbf{h} / \partial \mathbf{b}]=\left[\begin{array}{ll}
H_{a} & 0_{m \times 3}
\end{array}\right],
$$

since the attitude measurements are assumed not to depend explicitly on the gyro drifts. The Kalman gain matrix is given by

$$
K=\left[P_{a}(-) \quad P_{c}(-)\right]^{T} H_{a}^{T}\left[H_{a} P_{a}(-) H_{a}^{T}+R\right]^{-1},
$$

where $R$ is the covariance of the measurement white noise. The state update is given by

$$
\hat{\mathbf{x}}(+)=\hat{\mathbf{x}}(-)+K(\tilde{\mathbf{z}}-\hat{\mathbf{z}})=\hat{\mathbf{x}}(-)+K\left[\tilde{\mathbf{z}}-\hat{\mathbf{h}}-H_{u} \hat{\mathbf{a}}(-)\right],
$$

where $\tilde{\mathbf{z}}$ denotes the measured value and $\hat{\mathbf{z}}$ is the value predicted from the pre-update state estimate. The covariance update is

$$
P(+)=P(-)-\left[P_{a}(-) \quad P_{c}(-)\right]^{T} H_{a}^{T}\left[H_{a} P_{a}(-) H_{a}^{T}+R\right]^{-1} H_{a}\left[P_{a}(-) \quad P_{c}(-)\right] .
$$

\section{Reset}

The quaternion reset uses Eq. (17) with any of Eqs. (21); we have seen that the update is independent of the representation chosen. Resets can be performed after each measurement update, in which case the term $H_{a} \hat{\mathbf{a}}(-)$ in Eq. (55) is identically zero; but the reset is usually delayed until all the updates for a set of simultaneous measurements have been performed, for computational efficiency. It is imperative to perform a reset before beginning the next time propagation, however, to assure that $\hat{\mathbf{x}}(t)$ is zero at the beginning of the propagation, and thus to avoid the necessity of propagating $\hat{\mathbf{x}}(t)$ between measurements. The reset does not modify the covariance, since it neither increases nor decreases the total information content of the estimate; it merely moves this information from one part of the attitude representation to another.

\section{Quaternion Measurements}

Many modern star trackers track between 5 and to 50 stars simultaneously, match them to stars in an internal star catalogue, and compute their attitude as a inertially-referenced quaternion. ${ }^{33,34}$ The computation also produces an estimate of the $3 \times 3$ covariance of the attitude error angles. ${ }^{35.36}$ It is a simple matter to transform these quantities from the star tracker reference frame to the spacecraft frame to produce a quaternion "measurement" $\hat{q}$ and a $3 \times 3$ measurement covariance matrix $R$. The most convenient way to present the information in the measured quaternion to the Kalman filter is in terms of one of the threedimensional parameterizations of the estimated attitude error

$$
\delta q(\mathbf{a}=\tilde{\mathbf{z}}) \equiv \tilde{q} \otimes \hat{q}^{-1}(-) .
$$


The measurement model is simply

$$
\mathbf{h}(\mathbf{a})=\mathbf{a},
$$

so $H_{a}$ is the $3 \times 3$ identity matrix and $R$ is the covariance of this error angle. Since $\hat{\mathbf{h}}$ is identically zero from Eqs. (57) and (58), the state update is given by

$$
\hat{\mathbf{a}}(+)=\hat{\mathbf{a}}(-)+K_{a}[\tilde{\mathbf{z}}-\hat{\mathbf{a}}(-)]=\left(I_{3 \times 3}-K_{a}\right) \hat{\mathbf{a}}(-)+K_{a} \tilde{\mathbf{z}}
$$

and

$$
\hat{\mathbf{b}}(+)=\hat{\mathbf{b}}(-)+K_{b}[\tilde{\mathbf{z}}-\hat{\mathbf{a}}(-)],
$$

where

$$
K_{a}=P_{u}(-)\left[P_{u}(-)+R\right]^{-1},
$$

and

$$
K_{b}=P_{r}^{T}(-)\left[P_{a}(-)+R\right]^{-1} .
$$

It is important to use the same three-dimensional parameterization in the measurement model, Eq. (57), as is used in the reset. For example, if

$$
\mathbf{a}_{g}(t)=2\left[\tilde{q} \otimes \hat{q}^{-1}(-)\right]_{V} /\left[\tilde{q} \otimes \hat{q}^{-1}(-)\right]_{4},
$$

as in Eq. (35), then Eq. (21d) should be used for the reset. Alternatively, if the measurement is modeled as twice the vector part of the error quaternion, the reset should use Eq. (2lb) in place of Eq. (2ld). With this proviso, we see that when $R \ll<P_{a}(-)$, so that $K_{a}$ goes to the $3 \times 3$ identity matrix, we have $\hat{q}(+)=\tilde{q}$.

\section{SECOND ORDER FILTER}

Second-order terms in the nonlinear Kalman filter can become important when nonlinearities are significant relative to the measurement and process noise terms. According to Maybeck, ${ }^{4}$ a first-order filter with bias correction terms obtairis the essential benefit of a second order filter without the computational penalty of additional second moment calculations. This filter adds second-order corrections to the state propagation and measurement residual equations, but uses the EKF expressions for the covariance and gains.

\section{State and Covariance Propagation}

The state estimate is propagated by

$$
\dot{\hat{\mathbf{x}}}(t)=\left[\begin{array}{c}
\mathbf{f}(\hat{\mathbf{x}}(t), t)+\hat{\mathbf{b}}_{\mathrm{p}}(t) \\
\mathbf{0}
\end{array}\right],
$$

where the propagation bias correction is given by

$$
\left.\hat{\mathbf{b}}_{\Gamma}(t) \equiv \frac{1}{2} \sum_{i, j} \frac{\partial^{2} \mathbf{f}(\mathbf{x}, t)}{\partial x_{i} \partial x_{j}} P_{i j}(t)\right|_{\mathbf{x}=\hat{\mathbf{x}}(t)} .
$$

For the Gibbs vector parameterization, Eqs. (36), (41) and (63) give

$$
\hat{\mathbf{b}}_{\mathrm{r}}(t)=\frac{1}{4} P_{u}\left\{\hat{\boldsymbol{\omega}}(t)-2\left[\dot{\hat{q}}(t) \otimes \hat{q}^{-1}(t)\right]_{V}\right\}+\boldsymbol{\omega}_{\mathrm{c}}(t),
$$

where $\omega_{c}$ has components

$$
\left[\omega_{r}(t)\right]_{i} \equiv \frac{1}{2}\left\{\left[P_{c}(t)\right]_{k j}-\left[P_{r}(t)\right]_{j k}\right\}
$$


with $\{i, j, k\}$ a cyclic permutation of $\{1,2,3\}$. Including this second order term in Eq. (36) gives

$$
\begin{aligned}
\dot{\hat{\mathbf{a}}}_{g}(t) & =\left\{\frac{1}{2} \overline{\hat{\mathbf{\omega}}}(t) \otimes\left[\begin{array}{c}
\hat{\mathbf{a}}_{g}(t) \\
2
\end{array}\right]-\left[\begin{array}{c}
\hat{\mathbf{a}}_{g}(t) \\
2
\end{array}\right] \otimes \dot{\hat{q}}(t) \otimes \hat{q}^{-1}(t)\right\}_{V}+\frac{1}{4} P_{a}\left\{\hat{\boldsymbol{\omega}}(t)-2\left[\dot{\hat{q}}(t) \otimes \hat{q}^{-1}(t)\right]_{V}\right\} \\
& -\frac{1}{2}\left\{\frac{1}{2} \overline{\hat{\mathbf{\omega}}}(t) \otimes\left[\begin{array}{c}
\hat{\mathbf{a}}_{g}(t) \\
2
\end{array}\right]-\left[\begin{array}{c}
\hat{\mathbf{a}}_{g}(t) \\
2
\end{array}\right] \otimes \dot{\hat{q}}(t) \otimes \hat{q}^{-1}(t)\right\}_{4} \mathbf{a}_{g}(t)+\boldsymbol{\omega}_{c}(t) .
\end{aligned}
$$

The condition that $\hat{\mathbf{a}}_{g}(t)$ and $\dot{\hat{\mathbf{a}}}_{g}(t)$ are equal to zero is

$$
\left[\dot{\hat{q}}(t) \otimes \hat{q}^{-1}(t)\right]_{V}=\frac{1}{2}\left\{\hat{\boldsymbol{\omega}}(t)+\left[I_{3 \times 3}+\frac{1}{4} P_{a}(t)\right]^{-1} \boldsymbol{\omega}_{c}(t)\right\},
$$

It is shown in the Appendix that the factor of $\left[I_{3 \times 3}+\frac{1}{4} P_{a}(t)\right]^{-1}$ depends on the specific choice of the threedimensional parameterization of the rotation. Since $P_{u}(t)$ and $\boldsymbol{\omega}_{c}$ are both second order in the estimation errors, it is consistent with a second-order filter to replace $\left[I_{3 \times 3}+\frac{1}{4} P_{a}(t)\right]$ by the identity matrix, giving

$$
\dot{\hat{q}}(t)=\frac{1}{2}\left[\overline{\hat{\boldsymbol{\omega}}}(t)+\overline{\boldsymbol{\omega}}_{r}(t)\right] \otimes \hat{q}(t)=\frac{1}{2} \Omega\left(\hat{\boldsymbol{\omega}}(t)+\omega_{r}(t)\right) \hat{q}(t) .
$$

Time propagation between measurements is changed from the EKF by the addition of this second-order correction to the angular rate vector arising from the skew part of the covariance between the attitude errors and gyro drift bias errors. This is equivalent to the result obtained by Vathsal. ${ }^{17}$

\section{Measurement Update and Reset}

In the second-order filter, the predicted measurement $\hat{\mathbf{z}}$ has the form

$$
\hat{\mathbf{z}}=\hat{\mathbf{h}}+H_{a} \hat{\mathbf{a}}(-)+\hat{\mathbf{b}}_{\mathrm{m}}(t),
$$

with the measurement bias term given by ${ }^{4}$

$$
\left.\hat{\mathbf{b}}_{\mathrm{m}}(t) \equiv \frac{1}{2} \sum_{i, j} \frac{\partial^{2} \mathbf{h}(\mathbf{x}, t)}{\partial x_{i} \partial x_{j}} P_{i j}(t)\right|_{\mathbf{x}=\dot{\mathbf{x}}(t)} .
$$

Using Eq. (25) to expand Eq. (48) to second order in a gives

$$
\mathbf{h}\left(\mathbf{v}_{B}\right)=\mathbf{h}\left(\hat{\mathbf{v}}_{B}\right)+\left.\frac{\partial \mathbf{h}(\mathbf{v})}{\partial \mathbf{v}}\right|_{\hat{\mathbf{v}}_{B}}\left\{\left[\hat{\mathbf{v}}_{B} \times\right] \mathbf{a}+\frac{1}{2}\left(\mathbf{a}^{T}-a^{2} I_{3 \times 3}\right) \hat{\mathbf{v}}_{B}\right\}+\left.\frac{1}{2} \sum_{i, j=1}^{3} \frac{\partial^{2} \mathbf{h}(\mathbf{v})}{\partial v_{i} \partial v_{j}}\right|_{\hat{\mathbf{v}}_{B}}\left(\left[\hat{\mathbf{v}}_{B} \times\right] \mathbf{a}\right)_{i}\left(\left[\hat{\mathbf{v}}_{B} \times\right] \mathbf{a}\right)_{j}
$$

Inserting this into Eq. (70), using the symmetry of $P_{a}$ and the mixed second-order partial derivatives and the fact that the measurement does not depend explicitly on the gyro drift bias, gives

$$
\hat{\mathbf{b}}_{\mathrm{tu}}(t)=\left.\frac{1}{2}(\partial \mathbf{h} / \partial \mathbf{v})\right|_{\hat{\mathbf{v}}_{H}}\left[P_{a}-\left(\operatorname{tr} P_{a}\right) I_{3 \times 3}\right] \hat{\mathbf{v}}_{B}+\left.\frac{1}{2} \sum_{i, j=1}^{3}\left(\partial^{2} \mathbf{h} / \partial v_{i} \partial v_{j}\right)\right|_{\hat{\mathbf{v}}_{A}}\left(\left[\hat{\mathbf{v}}_{B} \times\right]^{T} P_{a}\left[\hat{\mathbf{v}}_{B} \times\right]\right)_{i j},
$$

where tr denotes the matrix trace. This result differs from the measurement bias found by Vathsal, whose computation ignored the quaternion norm constraint.

Now consider the special case that the attitude covariance $P_{a}$ is a multiple of the identity, $P_{a}=p_{a} I_{3 \times 3}$ for some scalar $p_{a}$. This case is of interest since a Kalman filter is often initialized with a large covariance of this form. We want to be sure that an unrealistically large covariance won't corrupt the update. In this case

$$
\hat{\mathbf{b}}_{\mathrm{tin}}(t)=p_{\mathrm{u}}\left\{-\left.(\partial \mathbf{h} / \partial \mathbf{v})\right|_{\hat{v}_{B}} \hat{\mathbf{v}}_{B}+\left.\frac{1}{2} \sum_{i j=1}^{3}\left(\partial^{2} \mathbf{h} / \partial v_{i} \partial v_{j}\right)\right|_{\hat{\mathbf{v}}_{i}}\left[\hat{v}_{B}^{2} \delta_{i j}-\left(\hat{\mathbf{v}}_{B}\right)_{i}\left(\hat{\mathbf{v}}_{B}\right)_{j}\right]\right\} \text {. }
$$


Now consider two different measurement models for a star tracker. The first is the usual model,

$$
\mathbf{h}(\mathbf{v})=\left[\begin{array}{l}
u_{1} / u_{3} \\
u_{2} / u_{3}
\end{array}\right]
$$

where

$$
\mathbf{u}=B \mathbf{v}_{B}
$$

is the unit vector to the star in the star tracker reference frame, which is rotated from the spacecraft body frame by the orthogonal transformation matrix $B$. The measurement sensitivity matrix is

$$
H_{a}=\frac{1}{u_{3}^{2}}\left[\begin{array}{ccc}
u_{3} & 0 & -u_{2} \\
0 & u_{3} & -u_{1}
\end{array}\right][\mathbf{u} \times] B
$$

The first term in Eq. (7.3) for the second order measurement bias vanishes, and the second term gives

$$
\hat{\mathbf{b}}_{\mathrm{m}}(t)=p_{\mathrm{u}}\left(1+\hat{h}^{2}\right) \hat{\mathbf{h}} \text {. }
$$

The second measurement model is linear:

$$
\mathbf{h}(\mathbf{v})=\left[\begin{array}{l}
u_{1} \\
u_{2}
\end{array}\right] .
$$

The measurement sensitivity matrix for this measurement model is

$$
H_{a}=\left[\begin{array}{lll}
1 & 0 & 0 \\
0 & 1 & 0
\end{array}\right][\mathbf{u} \times] B .
$$

In this case, the second term in Eq. (73) for $\hat{\mathbf{b}}_{\mathrm{um}}(t)$ vanishes, and the first term gives

$$
\hat{\mathbf{b}}_{\mathrm{m}}(t)=-p_{a} \hat{\mathbf{h}} \text {. }
$$

These two measurement models give measurement biases of the same order of magnitude but with opposite signs. For larger than usual star tracker initialization errors of 0.01 radians, or $0.573^{\circ}$, the correction to the predicted measurement is $0.1 \%$ of the leading term $\hat{\mathbf{h}}$.

Since the input to the Kalman Filter for a quaternion measurement is linear in the three-dimensional attitude parameter vector, the measurement bias $\hat{\mathbf{b}}_{\mathrm{w}}(t)$ is identically zero in this case. Also, since Eqs. (21) are exact to all orders of $\mathbf{a}$, the reset for the second-order filter is the same as the EKF.

\section{SUMMARY}

The major result of this paper is to clarify the relationship between the four-component quaternion representation of attitude and the three-component representation of attitude errors in the widely used estimator that has become known as the Multiplicative Extended Kalman Filter. We view this filter as based on an apparently redundant representation of the attitude in terms of a reference quaternion and a threevector specifying the deviation of the attitude from the reference. This apparent redundancy is removed by constraining the reference quaternion so that the expectation value of the three-vector of attitude deviations is identically zero. It is therefore not necessary to compute this identically zero expectation value. The basic structure of the Multiplicative Extended Kalman Filter follows from constraining the reference quaternion in this fashion: the reference quaternion becomes the attitude estimate, the three-vector becomes the attitude error vector, and the covariance of the three-vector becomes the attitude covariance. All these results are well known in practice, but the justification for using this mixed attitude representation has been unclear. Several different three-dimensional parameterizations give identical results in the linear Extended Kalman Filter and in a second-order filter, except in the reset step where they differ in third order in the measurement update. The second-order propagation and measurement biases are easily computed in this framework. 


\section{APPENDIX}

Instead of using the Gibbs vector, we will employ the vector part of the quaternion as in Eq. (2lb). With this parameterization, E.q. (36) is replaced by

$$
\mathbf{f}(\mathbf{x}(t), i) \equiv \dot{\mathbf{a}}_{q}(t)=\left\{\frac{1}{2} \overline{\mathbf{\omega}}(t) \otimes\left[\frac{\mathbf{a}_{q}(t)}{\sqrt{4-a_{q}^{2}(t)}}\right]-\left[\frac{\mathbf{a}_{q}(t)}{\sqrt{4-a_{q}^{2}(t)}}\right] \otimes \dot{\hat{q}}(t) \otimes \hat{q}^{-1}(t)\right\}_{V} .
$$

In the EKF approximation of Eq. (31),

$$
\mathbf{f}(\mathbf{x}(t), t)=-\hat{\mathbf{\omega}} \times \mathbf{a}_{q}(t)+\frac{1}{2} \sqrt{4-a_{q}^{2}} \Delta \boldsymbol{\omega}(t)-\frac{1}{2} \Delta \boldsymbol{\omega}(t) \times \mathbf{a}_{q}(t),
$$

which is the same as $\mathrm{E}_{\mathrm{l}}$. (37) through terms of second order in $\mathbf{a}$ and $\Delta \boldsymbol{\omega}$. Thus the EKF propagation and update equations are unchanged. Differentiating Eq. (A1) gives, with Eq. (63),

$$
\hat{\mathbf{b}}_{\mathrm{p}}(t)=-\frac{1}{8} \operatorname{tr} P_{a}(t)\left\{\hat{\boldsymbol{\omega}}(t)-2\left[\dot{\hat{q}}(t) \otimes \hat{q}^{-t}(t)\right]_{V}\right\}+\boldsymbol{\omega}_{r}(t),
$$

in place of Eq. (64), which changes Eq. (67) to

$$
\left[\dot{\hat{q}}(t) \otimes \hat{q}^{-1}(t)\right]_{V}=\frac{1}{2}\left\{\hat{\boldsymbol{\omega}}(t)+\left[1-\frac{1}{8} \operatorname{tr} P_{a}(t)\right]^{-1} \boldsymbol{\omega}_{r}(t)\right\} .
$$

Equations (67) and (A4) agree if and only if we ignore products of $P_{u}(t)$ and $\omega_{c}$.

\section{REFERENCES}

1. Kalman, R. E., "A New Approach to Linear Filtering and Prediction Problems," Transactions of the ASME, Series D, Journal of Basic Engineering, Vol. 82, pp. 35-45, 1960

2. Gelb, A., ed., Applicd Optimal Estimation, Cambridge, MA, the MIT Press, 1974

3. Stengel, R. F., Optimal Control and Estimation, New York, Dover Publications, Inc., 1994

4. Maybeck, P. S., Stochastic Models, Estimation, and Control, Arlington, VA, Navtech Book and Software Store, 1994, see especially Volume 2, pp. 224-225 and p. 249

5. Farrell, J. L., “Attıtude Determination by Kalman Filtering," Automatica, Vol. 6, pp. 419-430, 1970.

6. Pittelkau, M. E., "Spacecraft Attitude Determination Using the Bortz Equation," AAS Paper 01-310, AAS/AIAA Astrodynamics Specialist Conference, Quebec City, Quebec, August 2001

7. Stuelpnagel, J., "On the Parameterization of the Three-Dimensional Rotation Group," SIAM Review, Vol. 6, No. 4, pp. 422-430, 1964

8. Bar-Itzhack, I. Y., and Oshman, Y., "Attitude Determination from Vector Observations: Quaternion Estimation," IEEE Transactions on Aerospace and Electronic Systems, Vol. AES-21, No. 1, pp. $128-135,1985$

9. Lefferts, E. J., Markley, F. L., and Shuster, M. D., "Kalman Filtering for Spacecraft Attitude Estimation," Journal of Guidance, Control, and Dynamics, Vol. 5, No. 5, pp. 417-429, 1982

10. Bar-Itzhack, I. Y., Deutschmann, J., and Markley, F. L., "Quaternion Normalization in Additive EKF for Spacecraft Attitude Determination," AIAA Paper 91-2706, AIAA Guidance, Navigation, and Control Conference. New Orleans, LA, August 1991 
11. Deutschmann, J., Markley, F. L., and Bar-Itzhack, I., "Quaternion Normalization in Spacecraft Attitude Determination," Flight Mechanics/Estimation Theory Symposium, NASA Conference Publication 3186, Goddard Spice Flight Center, Greenbelt, MD, May 1992

12. Shuster, M. D., "The Quaternion in the Kalman Filter," Astrodynamics 1993, Advances in the Astronautical Sciences, Vol. 85, pp. 25-37, 1993

13. Toda, N. F., Heiss, J. L., and Schlee, F. H., "SPARS: the System, Algorithm, and Test Results," Proceedings of the Symposium on Spacecraft Attitude Determination, Aerospace Corp. Report TR-0066 (5306)-12, Vol. 1, pp. 361-370, Sept.-Oct. 1969

14. Murrell, J. W., "Precision Attitude Determination for Multimission Spacecraft," AIAA Paper 78-1248, AIAA Guidance and Control Conference, Palo Alto, CA, August 1978

15. Hamermesh, M., Group Theory and its Application to Physical Problems, Reading, MA, Addison-Wesley, 1962, Section 9-2

16. Curtis, M. L., Matrix Groups, $2^{\text {nd }}$ ed., New York, NY, Springer-Verlag, 1984, Chapter 5

17. Vathsal, S., "Spacecraft Attitude Determination Using a Second-Order Nonlinear Filter," Journal of Guidance, Control, and Dynamics, Vol. 10, No. 6, pp. 559-566, 1987

18. Wertz, J. R., ed., Spacecraft Attitude Determination and Control, Dordrecht, Holland, D. Reidel, 1978

19. Shuster, M. D., "A Survey of Attitude Representations," Joumal of the Astronautical Sciences, Vol. 41, No. 4, pp. 439-517, 1993

20. Euler, L., "Formulae Generales pro Trandlatione Quacunque Corporum Rigidorum," Novi Comment. Petrop., Vol. 20, pp. 189-207, 1775

21. Euler, L., "Problema Algebraicum Ob Affectiones Prorsus Singulares Memorabile," Novi Comment. Petrop., Vol. 15, Section 33, p. 101, 1770

22. Gauss, C. F., Werhe, Vol. VIII, pp. 357-362, Göttingen, Königliche Gesellschaft der Wisssenscaften, 1900

23. Rodrigues, O., "Des lois géométriques qui régissent les déplacements d'un système solide dans l'espace, et de la variation des coordonnées provenant de ces déplacements considérés indépendamment des causes qui peuvent les produire," Journal de Mathématiques, Vol. 5, pp. 380-440, 1840

24. Hamilton, W. R., "On Quaternions; or a New System of Imaginaries in Algebra," Philosophical Magazine, $3^{\text {rd }}$ Series, Vol. 25, pp. 489-495, 1844

25. Altmann, S. L., "Hamilton, Rodrigues, and the Quaternion Scandal," Mathematics Magazine, Vol. 62, No. 5, pp. 291-308, 1989

26. Gibbs, J. W., and Wilson, E. B., Vector Analysis, New York, NY, Scribner, 1901

27. Wiener, T. F., "Theoretical Analysis of Gimballess Inertial Reference Equipment Using DeltaModulated Instruments," D. Sc. Dissertation, Massachusetts Institute of Technology, March 1962

28. Marandi, S. R., and Modi, V. J., "A Preferred Coordinate System and the Associated Orientation Representation in Attitude Dynamics," Acta Astronautica, Vol. 15, No. 11, pp. 833-843, 1987

29. Gray, C. W., "Star Tracker/IRU Attitude Determination Filter," Guidance and Control 2001, Advances in the Astronautical Sciences, Vol. 105, Paper AAS 01-039 
30. Markley, F. L., “Attitude Representations for Kalman Filtering," AAS Paper 01-309, AAS/AIAA Astrodynamics Specialist Conference, Quebec City, Quebec, August 2001

31. Farrenkopf , R.L., "Analytic Steady-State Accuracy Solutions for Two Common Spacecraft Attitude Estimators," Journal of Guidance and Control, Vol. 1, No. 4, pp. 282-284, 1978

32. Markley, F. L. and Reynolds, R. G., "Analytic Steady-State Accuracy of a Spacecraft Attitude Estimator," Journal of Guidance, Control, and Dynamics, Vol. 23, No. 6, pp. 1065-1067, 2000

33. Chapel, J. D., and Kiessig, R., "A Lightweight, Low-Cost Star Camera Designed for Interplanetary Missions," Guidance' and Control 1998, Advances in the Astronautical Sciences, Vol. 98, Paper AAS $00-034$

34. Maresi, L., Paulsen, 'T., Noteborn, R., Mikkelsen, O. and Nielsen, R., "The TERMA Star Tracker for the NEMO Satellite," Guidance and Control 2000, Advances in the Astronautical Sciences, Vol. 104, Paper AAS 98-040

35. Shuster, M. D., "Maximum Likelihood Estimate of Spacecraft Attitude," Journal of the Astronautical Sciences, Vol. 37, No. 1, January-March 1989, pp. 79-88

36. Markley, F. L. and Mortari, D., "Quaternion Attitude Estimation Using Vector Observations," Journal of the Astronautical Sciences, Vol. 48, No. 2/3, April-Sept. 2000, pp. 359-380 